Editorial

\title{
Acknowledgement to Reviewers of Cosmetics in 2014
}

Cosmetics Editorial Office, MDPI AG, Klybeckstrasse 64, CH-4057 Basel, Switzerland

Published: 8 January 2015

The editors of Cosmetics would like to express their sincere gratitude to the following reviewers for assessing manuscripts in 2014:

\begin{tabular}{lll} 
Allinson, Nigel M. & Gonzalez, Salvador & Pao, Ching-I \\
Apone, Fabio & Green, Lawrence & Papanas, Nikolaos \\
Baldi, Alfonso & Haroutounian, Serkos A. & Pichardo-Geisinger, Rita O. \\
Ballarin, Barbara & Kalia, Yogeshvar N. & Pinazo, Aurora \\
Barba, Clara & Kapischke, Matthias & Quan, Taihao \\
Belmonte, J.C. Izpisua & Kim, Donguk & Rattan, Sureshi S. \\
BeMiller, James N. & Kim, Moon-Moo & Rodrigues, Francisca \\
Bilodeau, Diane & Klapper, Stephen R. & Rossi, Sergio \\
Breunig, H. G. & Koh, Jae Sook & Sadrieh, Nakissa \\
Bucay, V. W. & Kuck, Monika & Sakkas, V. \\
Cabral, Célia & Kustrin, Snezana & Sanchez, W. Y. \\
Cappiello, Achille & Labille, Jérôme & Sipp, Douglas \\
Chaintreau, Alain & Laganà, Aldo & Streker, Meike \\
Chirumbolo, Salvatore & Lee, Maw-Rong & Suschek, Christoph V. \\
Chung, Shin-Kyo & Lima, Cristovao F. & Taieb, Charles \\
Doudrick, Kyle & Mack Correa, M. C. & Touitou, Elka \\
Duarte, T. L. & Madonna, S. & Valente, Frederico \\
Failla, Cristina M. & Masaki, Hitoshi & Vávrová, Kateřina \\
Fasani, Elisa & McGhie, Tony K. & Vinardell, María Pilar \\
Feldman, Steven R. & Montenegro, Lucia & Viola, G. \\
Fukoura, Hirotaro & Morganti, P. & Wang, Moo-Chin \\
Gaikwad, Nilesh W. & Nanba, Daisuke & Zillich, Olesya V. \\
Goebel, Carsten & Nunn, Alistair V. W. & \\
\hline
\end{tabular}

(C) 2015 by the authors; licensee MDPI, Basel, Switzerland. This article is an open access article distributed under the terms and conditions of the Creative Commons Attribution license (http://creativecommons.org/licenses/by/4.0/). 